\title{
La empresa flexible como dispositivo de gobierno. Aportes de la Analítica de la Gubernamentalidad al estudio de las subjetividades laborales en América Latina*
}

\author{
The Flexible Enterprise as a Government Apparatus: \\ Contributions of the Analytics of Governmentality for \\ Study of Labor Subjectivities in Latin America
}

Recibido: 12 de noviembre de 2014 | Aceptado: 7 de agosto de 2015

\author{
ANTONIO STECHER ** \\ Universidad Diego Portales, Santiago, Chile
}

doi :10.11144/Javeriana.upsy14-5.efdg

Para citar este artículo: Stecher, A. (2015). La empresa flexible como dispositivo de gobierno. Aportes de la Analítica de la Gubernamentalidad al estudio de las subjetividades laborales en América Latina. Universitas Psychologica, 14(5), 1779-1794. http://dx.doi.org/10.11144/Javeriana.upsy14-5.efdg

Artículo de reflexión El presente artículo se ha elaborado en el marco de un proyecto de investigación financiado por el Fondo Nacional de Desarrollo Científico y Tecnológico del gobierno de Chile (FONDECYT). Proyecto Fondecyt № 11130095 :

"Procesos de construcción de identidad en el trabajo en el Chile actual: el caso de los trabajadores de tienda de grandes empresas del Retail. Aportes empíricos y conceptuales al debate sobre trabajo e identidad en América Latina".

** Facultad de Psicología. La correspondencia de este artículo debe ir dirigida a Antonio Stecher, Facultad de Psicología, Universidad Diego Portales, Grajales 1898, Santiago, Chile. Correo electrónico: antonio.stecher@udp.cl
RESUMEN

Se aboradán los aportes de la Analítica de la Gubernamentalidad desarrollada por Foucault para interrogar críticamente las transformaciones laborales y subjetivas ocurridas en América Latina, en el contexto del capitalismo flexible. Se discute el modo como la empresa flexible y su discurso neomanagerial puede ser conceptualizado como un dispositivo de gobierno en que se entrelazan particulares juegos de verdad, relaciones de poder y vectores de subjetivación. Se analizan cuatro rasgos de la racionalidad de gobierno de la empresa flexible -capital humano, incorporación de la vida y la subjetividad social al proceso productivo, tecnologías posdisciplinarias de control, nuevo ideal de trabajador flexible-, destacando los modos de subjetivación laboral que promueven, así como sus afinidades con las lógicas de la gubernamentalidad neoliberal.

Palabras clave

subjetividad; trabajo; gubernamentalidad; empresa flexible; Foucault

\footnotetext{
A B S T R A C T

We discuss the contributions of the analytics of governmentality developed by Foucault to critically examine transformations in work and in labor subjectivities in Latin America, in the context of flexible capitalism. The way the flexible enterprise and neomanagerial discourse can be conceptualized as a government apparatus, in which particular games of truth, power relations and ways of subjectivation are intertwined, is discussed. We present 4 traits of government racionality of the flexible enterprise - Human capital, incorporating social life and subjectivity in the production process, postdisciplinary control technologies, new ideal of flexible worker- highlighting ways of labor subjectivation that promote and their affinities with the logic of neoliberal governmentality.

Keywords

subjectivity; work; governmentality; flexible enterprise; Foucault
} 


\section{Introducción}

Uno de los principales desafíos para la psicología social del trabajo en América Latina es el desarrollo de marcos conceptuales que permitan analizar e interrogar críticamente los actuales procesos de producción de subjetividades laborales. Esto, en el contexto de la inserción de los países de la región al nuevo régimen de acumulación capitalista (flexible, global, informacional, en red) y a su matriz de regulación sociopolítica neoliberal o de liberalismo avanzado (Amin, 1994; Castells, 2001, 2005; De la Garza, 2000; Harvey, 1998). Como ha sido ampliamente documentado, dicha dinámica mundial de reestructuración capitalista y reconfiguración de las sociedades modernas (tránsito de la modernidad organizada o sólida a la modernidad tardía o líquida) ha conllevado en las últimas tres décadas fuertes procesos de reestructuración productiva, modernización empresarial y flexibilización laboral en América Latina, los cuales adquieren características diferenciadas según los particulares contextos nacionales, sectores económicos, industrias, tipos de empresa u organización (pública/privada, grande/ pequeña, formal/informal, etc.), entre otros (De la Garza, 2000; Neffa, 2003; Stecher \& Godoy, 2014). Dichos procesos han transformado fuertemente los escenarios laborales y las condiciones y experiencias de trabajo de vastos sectores de la población, dando lugar a nuevas y singulares configuraciones de subjetividad laboral. Esto es, a nuevas modalidades de experimentar e interpretar la experiencia laboral y de constituirse como sujeto laboral de un cierto tipo con particulares modos de pensar, sentir y actuar respecto a sí mismo, los otros y el mundo en tanto trabajador (Battistini, 2004; Soto, 2008; Stecher, 2013; Tittoni \& Nardi, 2011).

Buscando contribuir al debate y enriquecimiento de perspectivas de análisis que permitan interrogar críticamente dichos procesos de reconfiguración de las subjetividades laborales en América Latina, este artículo presenta y discute el marco de la Analítica de la Gubernamentalidad formulada por Michel Foucault $(2006,2007)$ en sus cursos de finales de los años 70 en el Collège de France, y continuada posteriormente por un conjunto de autores anglófo- nos que dieron forma a los denominados Estudios de la gubernamentalidad (Castro-Gómez, 2010; Dean, 2008; Miller \& Rose, 2008; Rose, 1997; Rose \& Miller, 1992; Vázquez, 2005). Dentro de este vasto campo, el argumento de este artículo pondrá el foco y se limitará a dar cuenta de cómo los conceptos de gubernamentalidad, racionalidades de gobierno y gubernamentalidad neoliberal, constituyen claves fecundas para analizar a la nueva empresa flexible y sus principios neomanageriales de gestión, así como a las nuevas formas de subjetividad laboral que se producen en su seno.

Con ese objetivo, se ha organizado el artículo de la siguiente manera. En primer lugar, se presentan muy brevemente algunos elementos básicos de la analítica y la historia de la gubernamentalidad. En segundo lugar, se discuten las tres grandes familias de gubernamentalidad posibles de distinguir en el itinerario histórico de las sociedades modernas, relevando las características de la actual gubernamentalidad neoliberal y del sujeto empresario de sí. En tercer lugar, se examina el modo en que la empresa flexible y su racionalidad neomanagerial, puede ser conceptualizado como un dispositivo de gobierno que promueve un particular tipo de subjetividad laboral. En cuarto lugar, se analizan cuatro ejes que definen la especificidad de la racionalidad de gobierno de la empresa flexible, visibilizando las articulaciones de cada uno de ellos con los procesos de producción de subjetividades laborales. Por último, en las reflexiones finales, se destacan ciertas consideraciones respecto al uso de la analítica de la gubernamentalidad en la investigación psico-socio-laboral en América Latina, y se pone de relieve el potencial crítico y transformador de dicha perspectiva.

\section{Historia y analítica de la gubernamentalidad}

La historia de la gubernamentalidad desarrollada por Foucault $(2006,2007)$ en los seminarios de 1978 "Seguridad, Territorio y Población" y "El nacimiento de la Biopolítica" de 1979, busca dar cuenta del particular régimen de poder que emerge y sirve de base a la formación del Estado moderno-liberal a 
partir del siglo XVIII. Se trata de un régimen de poder (el Estado de gobierno o la gubernamentalización del Estado) que adquiere primacía sobre otros mecanismos de poder jurídicos o disciplinarios, y que se caracteriza por tener como foco principal la gestión de la vida de la población (biopolítica), a través de los conocimientos entregados por el saber económico liberal, y que opera con base en dispositivos de seguridad o mecanismos de regulación (Foucault, 2006; Ramos, 2012).

Lo distintivo de los dispositivos de seguridad, o del liberalismo entendido como tecnología de gobierno que marca el surgimiento y despliegue, hasta nuestros días, de la modernidad ${ }^{1}$, es que estos no se basan en la autorización/prohibición vertical de ciertas actividades (mecanismos jurídicos, de soberanía) ni en la normación de los comportamientos con base en códigos predefinidos y fuertemente prescriptivos a los que los individuos deben ajustarse (mecanismos disciplinarios). Se basan, más bien, en la regulación, anticipación y gestión de eventos y fenómenos, con base en los conocimientos de los saberes de las disciplinas científicas y asumiendo el principio de no intervenir sus regularidades y leyes internas, así como en la creación de ciertos marcos que favorecen que los mismos agentes opten libremente por ciertos tipos de conducta (CastroGómez, 2010; Ramos, 2012). El desplazamiento central, que es necesario advertir en este análisis de Foucault de la gubernamentalidad, es el tránsito, tanto conceptual como histórico, desde un modelo que entiende las relaciones de poder en términos de

1 Entendemos el término "modernidad" como una categoría que da cuenta de un particular período histórico que se inicia hacia fines del siglo XVIII y se extiende hasta nuestros días. Como señala Castro (2011), este sentido de "modernidad" es el que aparece en obras de Foucault como Historia de la Locura en la Edad Clásica, Las Palabras y las Cosas y Vigilar y Castigar. Si bien en estas obras el foco está en una determinación epistémica de la modernidad (en ruptura con la Época Clásica), es posible considerar ese mismo criterio y entenderla como aquel período histórico, de fines del siglo XVIII hasta nuestros días, caracterizado por el surgimiento, progresiva expansión y consolidación de la gubernamentalización del Estado y su racionalidad de gobierno liberal (véase la nota 4). La opción por este uso de la noción de modernidad, en tanto período histórico en que se despliegan las sociedades modernas y su racionalidad de gobierno liberal (divididas, ver más adelante, en tres etapas) permite establecer un diálogo fecundo entre la obra de Foucault, en particular su analítica de la gubernamentalidad, y la sociología de la modernidad y del trabajo (Wagner, 1997). dominación a uno basado en la noción de gobierno, donde este último es entendido como la conducción de la conducta de personas con capacidad de libre elección y decisión, hacia ciertos fines y resultados, incluyendo tanto el conducir y guiar a otros, como el conducirse a si mismo en un campo de acciones posibles (Foucault, 2001). De este modo, el gobierno no es opuesto a la libertad, sino que produce y presupone agentes libres cuya conducta se busca conducir hacia ciertos fines, interviniendo en las condiciones del entorno donde toman opciones y modulando las formas en que se autogobiernan (Binkley, 2014) 2 . Como escribe Castro-Gómez (2010):

Y si las tecnologías de gobierno presuponen ya de entrada la capacidad de acción de los individuos, es decir su libertad, queda entonces claro que la meta de estas tecnologías es la autorregulación: lograr que el gobernado haga coincidir sus propios deseos, decisiones, esperanzas, necesidades y estilos de vida con objetivos gubernamentales fijados de antemano. Por eso gobernar no significa obligar que otros se comporten de cierta forma (y en contra de su voluntad), sino lograr que esa conducta sea vista por los gobernados mismos como buena, honorable, digna $y$, por encima de todo, como propia, como proveniente de su libertad (...) [hay una] nueva concepción del poder como gobierno sobre acciones. No se interviene directa e inmediatamente sobre los otros (reprimiendo sus acciones de modo violento), sino sobre el campo posible de sus acciones. No se busca, entonces, anular la libertad de los sujetos, sino conducirla, y esto a través de unas tecnologías específicas. (pp. 43-44)

Siguiendo a Miller y Rose (2008), Castro-Gómez (2010) y Rose (2003) es posible señalar que llevar a cabo una analítica de la gubernamentalidad en un determinado momento histórico, contexto y ám-

2 De este modo, el análisis de la gubernamentalidad exige atender a la zona donde se articulan tanto las micro tecnologías del yo, a partir de las cuales los individuos intervienen sobre sí mismos y se subjetivan y gobiernan de un cierto modo en pos de ciertos objetivos, como las macro tecnologías vinculadas a los mecanismos de seguridad y al gobierno (desde el Estado y las autoridades) de la población o ciertos grupos dentro de ella (Binkley, 2014; Dean, 2008). 
bito social particular de las sociedades modernas, supone interrogarse por la racionalidad específica de gobierno (objetivos especiales para la orientación de la conducta, medios y estrategias), que articula y ensambla en una cierta dirección un conjunto de prácticas discursivas y no discursivas en un específico dominio o mundo social (la industria, la escuela, el consumo, la familia, etc.). En términos más precisos, orientar una investigación empírica desde dicha perspectiva de análisis exige abordar tres ejes (genealógico, arqueológico y de la relación del sujeto consigo mismo) y plantearse, para cada uno de ellos, un conjunto de interrogantes. Se presentan brevemente estos tres ejes y se ilustran las principales interrogantes asociadas a los mismos, tomando como ejemplo la fuerte expansión de los principios flexibilizadores del new management en las grandes empresas y organizaciones del capitalismo contemporáneo (Boltanski \& Chiapello, 2002; Zangaro, 2011).

El primer eje se refiere a la necesidad de llevar a cabo (i) un análisis genealógico del modo en que una situación específica se configuró como problemática para ciertas agencias y autoridades, dando paso a intervenciones y técnicas (dimensión tecnológica) para conducir la conducta de colectivos e individuos hacia objetivos puntuales considerados deseables; objetivos locales que eventualmente resuenan con determinados fines políticos, morales, sociales más amplios de una cierta racionalidad política. Así, por ejemplo, es posible interrogarse, ¿de qué manera y como resultado de qué procesos ciertas lógicas y prácticas de gestión empresarial burocrática-fordista comienzan a aparecer como problemáticas para grupos definidos o autoridades nacionales y empresariales, emergiendo la necesidad de conducir la conducta de los cuadros gerenciales y profesionales dentro de las organizaciones hacia nuevas prácticas de gestión flexible, nuevos objetivos y lógicas productivas?, ia través de qué mecanismos, técnicas y procedimientos concretos y específicos (espacios de formación en las escuelas de negocios, mecanismos de incentivos, formas de control) articulados en una cierta estrategia, se ha buscado (re)conducir en forma eficaz la conducta de los cuadros gerenciales y profesionales que adminis- tran las empresas y organizaciones?, ¿de qué modo dichos nuevos principios de gestión y nuevos objetivos locales para la acción empresarial resuenan y son afines con la racionalidad política neoliberal más amplia de la modernidad contemporánea?

El segundo eje se refiere a la necesidad de realizar (ii) un análisis arqueológico de los discursos o juegos de verdad que configuran, hacen inteligible y legitiman epistémicamente la problemática, los objetivos de gobierno, las tecnologías y las autoridades. Acá, por ejemplo, sería posible preguntarse por icuáles discursos o juegos de verdad han configurado discursivamente y dotado de inteligibilidad y legitimidad epistémica a la representación del carácter anacrónico, ineficaz y negativo de las formas burocráticas y fordistas de administración, asî como del carácter virtuoso, moderno e ineludible de las nuevas lógicas flexibles del new management, dotando de verosimilitud cognitiva a los objetivos de conducción de la conducta que definen la nueva racionalidad de gobierno del mundo empresarial y de las organizaciones?, icuáles son los sujetos (economistas) e instituciones (escuelas de economía, programas de MBA) que aparecen como autoridades para decir la verdad sobre las mejores formas de gestión empresarial y para implementar las tecnologías de gobierno específicas a las que se asocian?

El tercer eje alude a la importancia de llevar a cabo (iii) un análisis de la ética o del modo de relación del sujeto consigo mismo (subjetivación) que es alentada en un escenario social específico por la particular racionalidad de gobierno y sus juegos de verdad (asujetamiento); esto es, un análisis de los procesos de subjetificación o de producción de subjetividades de un cierto tipo en el marco de ciertas relaciones históricas de saber/poder ${ }^{3}$. ¿Qué nuevos modelos o

3 Siguiendo a Rose (2003) es importante hacer la siguiente distinción terminológica. Por subjetificación se alude a los procesos de producción de subjetividad, esto es, de sujetos de un cierto tipo. Los procesos de subjetificación, a su vez, implican siempre modalidades de asujetamiento o sujeción a ciertos juegos de verdad y diagramas de poder que objetivan a los agentes humanos como sujetos de un cierto tipo; así como procesos de subjetivación que dan cuenta del particular modo de relación con uno mismo, del trabajo de uno sobre sí mismo en pos de cierto telos, que se configura en el crisol de esos mismos juegos de verdad y formas de autoridad. Dicho en otros términos, la subjetividad es el producto de procesos históricos y situados de subjetificación a través de los 
ideales de empresario, gerente y trabajador y qué modalidades específicas de relación de uno consigo mismo, son promovidas como parte de los esfuerzos de (re)conducir la conducta de los individuos dentro de las empresas y organizaciones, buscando favorecer la autorregulación y el alineamiento de las aspiraciones y decisiones libres de cada profesional y gerente con los objetivos definidos y considerados deseables por las autoridades de la esfera económica y empresarial?, icuáles son las conexiones que es posible identificar entre el tipo de subjetividad o relación consigo mismo que favorece entre los cuadros profesionales y gerenciales la racionalidad de gobierno neomanagerial y ciertos objetivos políticos, sociales, morales más amplios propios de la racionalidad política neoliberal situada en una escala mayor? (Rose, 2003).

Es desde esta trípode analítica (autoridad/poder, juegos de verdad, subjetivación) que la analítica de la gubernamentalidad incorpora los tres grandes momentos o perspectivas de análisis de la obra de Foucault (arqueología, genealogía, ética), ofreciendo un marco conceptual fecundo para analizar los procesos de producción de subjetividades (subjetificación) en diferentes campos de relaciones sociales al interior de las sociedades modernas (Dreyfus \& Rabinow, 2001; Miller \& Rose, 2008; Zangaro, 2011). Se trata de una perspectiva anitiesencialista, antidualista y crítica que permite visibilizar el modo en que las formas de subjetividad presentes en un particular campo social y escenario sociohistórico están vinculadas a las racionalidades de gobierno imperantes. Racionalidades cuya operación de conducción de la conducta, a través de diversas técnicas y en el marco de ciertos juegos de verdad, se basa, justamente, en tratar de modelar de cierta forma los deseos, aspiraciones, intereses y creencias de los individuos para que estos elijan, en tanto agentes libres, conducirse a sí mismos de un particular modo en un campo posible de acciones (Dean, 2008). Como ha escrito Paul du Gay (1996):

\section{(...) el gobierno opera a través de los sujetos. (...).}

cuales, y mediante lógicas de asujetamiento y subjetivación, los agentes humanos se constituyen en un particular tipo de sujeto.
Las formas de poder funcionan pues construyen y mantienen las formas de subjetividad más apropiadas a un tipo dado de racionalidad de gobierno. Las Subjetividades son constituidas y están ligadas a particulares formas de poder a través de diversas técnicas, saberes y prácticas inmanentes a esa forma de poder. Así el poder funciona en y a través de la subjetividad. Diferentes racionalidades de gobierno (...) están ligadas a concepciones y atributos de aquellos a quienes se gobierna (...) involucran la construcción de particulares formas de ser para los individuos. (p. 55)

\section{Formas históricas de gubernamentalidad y racionalidad neoliberal}

Si bien existe una continuidad histórica en la gubernamentalidad, en tanto racionalidad de gobierno que articula las prácticas y configuraciones sociales que constituyen al Estado moderno liberal desde el siglo XVIII hasta nuestros días ${ }^{4}$, es posible distinguir dentro de ella tres grandes familias o períodos (Dean, 2008; Miller \& Rose, 2008): el liberalismo clásico, el liberalismo social y el liberalismo avanzado o neoliberalismo. Estas tres familias de gubernamentalidad comparten la primacía de los mecanismos de seguridad ${ }^{5}$ para el gobierno de la población, grupos e instituciones, así como el presupuesto y la producción de la capacidad de autogobierno y libertad de los individuos, la cual es gestionada a través de la intervención en las condiciones o el medio en que los agentes calculan y

4 La noción de gubernamentalidad puede ser entendida, en un sentido acotado, como la lógica o racionalidad de gobierno que define la especificidad histórica de las prácticas de los Estados modernos liberales. También puede ser entendida, en un sentido más amplio y abstracto, como un concepto que busca iluminar el tipo de lógica o racionalidad que organiza un determinado campo de conducción de la conducta, pudiéndose hablar de gubernamentalidad más allá del campo de las tecnologías liberales de gobierno. Se ha optado en este artículo por el primero de esos dos usos (ambos presentes en la obra de Foucault) (Senellart, 2006).

5 Hablar de la primacía de los mecanismos de seguridad no quiere decir que los mecanismos de soberanía o disciplinarios dejen de existir, sino más bien que estos tienen un rol más acotado en ciertos ámbitos de la vida social y que, sobre todo, se acoplan con y operan "al servicio del" vector predominante dado por los mecanismos de seguridad. 
eligen por sí mismos sus cursos de acción (Binkley, 2014; Ramos, 2012). Se describirá brevemente cada una de estas tres familias, con un mayor foco y atención en la gubernamentalidad neoliberal, en tanto racionalidad política contemporánea en la cual es posible situar los procesos de reestructuración productiva, modernización empresarial, flexibilización y producción de nuevas subjetividades laborales que son el centro de interés de este artículo.

a) El liberalismo clásico emerge en el siglo XVIII y se basa en un gobierno económico que reconoce un conjunto de esferas (mercado, sociedad civil, vida privada) como exteriores al Estado, con su propia autonomía y legalidad, la que debe ser respetada y no intervenida directamente. Se trata de un gobierno económico basado en la promoción de los mecanismos naturales del mercado descritos por la ciencia económica, lo que exige la producción de la libertad (de trabajo, de comercio, de propiedad, de participación) de los agentes (homo economicus) y la no intervención o limitación de la acción del Estado para no perturbar las leyes internas y favorecer la autorregulación de los procesos económicos (mercado), biológicos (población) y culturales (sociedad civil) (Miller \& Rose, 2008; Vázquez, 2005).

b) El liberalismo social que empieza a gestarse en el último tramo del siglo XIX, se legitima como forma predominante de gobierno a partir de 1930 en el contexto de la grave crisis económica del capitalismo, y alcanza su máxima consolidación en el marco del llamado Estado social de bienestar en el período de la posguerra entre 1945 y 1975. El liberalismo social surge como una reacción a la insuficiencia de la lógica del laissez faire del liberalismo clásico, para dar respuesta a un conjunto de dinámicas (hacinamiento, pauperismo, insalubridad, migración, crecimiento urbano, amenaza de la revolución comunista) producidas por la propia modernización y que amenazaban la viabilidad de la modernidad liberal capitalista. Surge así, en el contexto de lo que se denominó el despunte de la cuestión social, la necesidad de ampliar y fortalecer las modalidades de actuación e intervención estatal en la vida económica y social (Vázquez, 2005). Este nuevo gobierno social supuso el despliegue de amplios mecanismos de protección y seguridad, enmarcados en el saber experto de las disciplinas científicas, orientados a cubrir un conjunto de necesidades básicas (salud, educación, vejez, vivienda) de los individuos para alentar la integración y cohesión social. Se trató de mecanismos basados en lógicas de solidaridad e interdependencia mutua, que se alejaban parcialmente de la figura del homo economicus responsable por sí mismo de la gestión de los riesgos, y que instituían la figura del "ciudadano social" como un sujeto de necesidades, gobernado a través de su inserción y dependencia en una tupida malla de reglamentaciones y categorizaciones colectivas (Dean, 2008; Ramos, 2012; Vázquez, 2005).

c) El neoliberalismo o liberalismo avanzado surge como una respuesta al gobierno social y a la injerencia del Estado, caracterizándose por lo que Foucault (2007) denominó como una "Fobia al Estado". Primero, como formulación teórica en el ordoliberalismo alemán de los años 30 y en el neoliberalismo de la escuela económica de Chicago de los años 50 y 60 , y posteriormente como forma de gubernamentalidad predominante en la vida social a partir de 1980; el neoliberalismo (o liberalismo avanzado) debe entenderse como una nueva modalidad de racionalidad política liberal. Lo distintivo de esta es su rechazo absoluto a la injerencia y planificación estatal y a la dependencia de los individuos respecto a los mecanismos de protección social, así como la reorganización de todas las relaciones sociales bajo la forma empresa (Foucault, 2007; McNay, 2009). Esta particular forma de racionalidad de gobierno -que emerge como una respuesta a un conjunto de problematizaciones y limites a los que se confronta el capitalismo fordista-keynesiano en la década de los 70, producto de procesos tales como la globalización económica, la revolución tecnológica, los mayores niveles de educación e individualización, la crisis del proceso de acumulación, etc. - no es una mera reedición del liberalismo clásico. Ya no se trata solo de un Estado que reconoce y respeta las leyes del mercado, y alienta el intercambio de mercancías entre los agentes individuales entendidos como homo economicus (Foucault, 2007). Se trata de un Estado que activamente reorganiza y sostiene el funcionamiento de la totalidad de la vida social 
bajo los principios y la dinámica de la competencia, el riesgo y la empresarización; incluyendo esto un proceso de empresarización de la vida, las experiencias y las propia subjetividad (Foucault, 2007; McNay, 2009; Rose 2003).

El neoliberalismo no implica una desaparición del Estado ni de "lo social", sino una reorganización de su gobierno a través de "la proliferación organizada de diferencias individuales en una matriz económica (...) de la multiplicación de las libertades de elección” (McNay, 2009, p. 59), de fuertes procesos de descentralización, desjerarquización y conectividad en nuevas escalas globales, y de nuevas formas de regulación (ranking, auditorias, estándares, cumplimiento de metas, exigencias de calidad y transparencia, etc.) orientadas por lógicas mercantiles y de competencia (Miller \& Rose, 2008). La noción de "empresario de sí" - de un modo equivalente a lo ya planteado del "homo economicus" y el "ciudadano social"- busca dar cuenta de las particulares modalidades de asujetamiento y subjetivación que caracterizan a los procesos de subjetificación bajo las coordenadas históricas de la gubernamentalidad neoliberal. Se trata de un perfil de sujeto fuertemente individualizado, emprendedor, responsable de su destino, que vive expuesto al riesgo y que debe ser capaz de reinventarse permanentemente. Un sujeto que debe autorregularse y gestionarse a sí mismo en los distintos mercados en los que participa, que valora fuertemente la satisfacción individual vía el consumo de bienes, servicios y experiencias, que rehúsa y desconfía de las jerarquías y autoridades institucionales, afirmando siempre el valor de la libertad, el bienestar subjetivo, la felicidad personal y la autorrealización personal, orientado por la búsqueda estratégica de desarrollarse a sí mismo, potenciarse, superar o vencer sus propios límites, ampliar sus competencias y cualidades -entendidas como un capital humano- de modo de diferenciarse, encontrar nichos de oportunidad y destacarse en un espacio social marcado por la competencia continua con distintos actores (Binkley, 2014; Rose, 2003). Este "empresario de sí" que afirma su libertad de elección, que se propone permanentemente nuevos proyectos de superación y que se orienta por el deseo de "conducir su propia existencia como un proyecto para la maximización de la calidad de vida" (Rose, 2003, p. 244), implica una ruptura con las lógicas de regulación del liberalismo social donde la fuerte prescripción normativa y los mecanismos disciplinarios -ensamblados en lógicas seguritarias- jugaron un rol importante en amplios dominios e instituciones sociales: la familia, la escuela, la fábrica. Se trata, así, del momento de máxima expansión de las tecnologías de gobierno liberales que caracterizan la modernidad, y que se basan en la producción y afirmación de la "libertad" de elección de cada individuo pensado, bajo la lógica neoliberal, como un empresario de sí mismo (Burchel, 1996).

\section{La empresa flexible y el nuevo management como dispositivo de gobierno}

Uno de los aportes de la historia y la analítica de la gubernamentalidad al campo de los estudios críticos del trabajo y las organizaciones en América Latina es la posibilidad que nos brinda de analizar a las grandes empresas de alta productividad, pertenecientes al estrato moderno y formal de la economía y conectadas a los circuitos económicos globales, como nuevos dispositivos de gobierno organizados en torno a los principios de organización y gestión flexible del nuevo management (Weiskopf \& Munro, 2011; Zangaro, 2011). Si bien las grandes empresas no representan, en ningún caso, a la totalidad de los heterogéneos y desiguales mundos del trabajo de los países de la región -donde el $48 \%$ de los trabajadores está inserto en el sector informal (Tokman, 2011) -, juegan un rol central en términos de productividad, generación de empleo asalariado y modelo de lo que es considerado y promovido como prácticas de gestión modernas. De este modo, constituyen un ámbito privilegiado para analizar los procesos de reestructuración productiva, modernización empresarial y flexibilización laboral, ocurridos en las últimas décadas en los distintos países de la región, así como el modo en que dichos procesos han conllevado importantes transformaciones en las subjetividades laborales. 
Al hablar de empresa flexible nos referimos a un tipo ideal que da cuenta de una particular articulación de elementos tecno-socio-productivos, a través de los cuales se despliega una nueva y particular modalidad de racionalización capitalista del trabajo. Se trata del prototipo ideal de unidad productiva en el contexto del nuevo régimen de acumulación (flexible, en red, informacional) y de regulación sociopolítica (neoliberal) que caracteriza al capitalismo contemporáneo. En términos muy esquemáticos y enumerativos la empresa flexible o posfordista, que se define en gran medida por oposición a la gran industria taylorista-fordista y burocrática del capitalismo industrial, se caracteriza, según autores como Castells (2001), Neffa (2003), Ramos (2009), Sennett (2006) y Vallas (1999) por:

a) nuevas formas de organización de la producción y gestión empresarial: empresa red, descentralización productiva, externalización y creación de redes empresariales, principios de mercantilización y competencia interna entre unidades/trabajadores de la misma empresa, sistemas de producción just in time orientados desde la demanda del cliente, exigencias de calidad total, ideal de empresa esbelta o magra que reduce los tiempos muertos de trabajo y produce el máximo valor con el mínimo de recursos, innovación continua, producción versátil, sistemas de evaluación constante y de remuneración fuertemente individualizados y ligados al cumplimiento de metas y objetivos.

b) nuevas modalidades postayloristas, toyotistas o flexibles de organización del trabajo: polivalencia y polifuncionalidad, equipos de trabajo semiautónomos, círculos de calidad, rotación y ampliación de puestos, movilización de destrezas cognitivas y emocionales, control normativo basado en la autorregulación, intensificación del trabajo, responsabilización individual y exigencia de alta implicación y disponibilidad permanente del trabajador, centralidad del cliente como agente de control del proceso de trabajo, exigencias permanentes de capacitación y aprendizaje continuo, fuerte uso de formas de flexibilidad temporal y salarial.

c) Procesos de flexibilización del empleo y cambio en las relaciones laborales: procesos de individualización, (re)mercantilización y des-colectivización de las relaciones laborales, debilitamiento de la organización sindical como actor sociopolítico, uso creciente de formas flexibles, atípicas, inciertas y habitualmente precarias de empleo (contratos a plazo fijo, por obra/faena o proyectos, temporales, de practicante, trabajadores suministrados, subcontratados, jornadas part-time, por honorarios, etc.).

d) Procesos de innovación tecnológica: uso de nuevas tecnologías computacionales de información y comunicación, soporte de las nuevas formas de gestión y organización en red que permiten acelerar, automatizar, adaptar e interconectar a escala local y global procesos productivos de bienes y servicios, y que juegan un rol central en la difuminación de los clásicos límites entre tiempos y espacios laborales y no laborales.

e) Nuevos discursos, saberes y cultura laboral: expansión desde los cuadros gerenciales de una cultura laboral de corte neomanagerial y posfordista que instituye nuevos valores y sentidos de la experiencia laboral vinculados al cambio, la flexibilidad, los proyectos, la innovación, la gestión del riesgo y la incertidumbre; así como nuevos ideales de sujeto laboral vinculados a la figura del trabajador flexible y emprendedor (Sennett, 2006).

Desde la perspectiva de la analítica de la gubernamentalidad, y poniendo el foco no ya en las racionalidades políticas de la sociedad, sino en un ámbito acotado y local del mundo social como lo es el trabajo, es posible interrogar a la empresa flexible como un particular dispositivo organizado en torno a una específica racionalidad de gobierno. Esto es, como un específico entramado heterogéneo de prácticas discursivas y no discursivas, articuladas por una específica racionalidad práctica o programa de gobierno que define ciertos objetivos, medios y estrategias empresariales, y configura un espacio de conducción de la conducta de los sujetos laborales a través de la estructuración de un campo posible de acciones (Rabinow \& Rose, 2001). En tanto dispositivo, la empresa flexible da cuenta de una estrategia histórica específica orientada, a través de nuevas lógicas de gestión y organización productiva, a afrontar los límites alcanzados por la industria taylorista-fordista y relanzar el proceso de acumulación capitalista en el marco de los parámetros del 
nuevo capitalismo (global, informacional, en red, flexible) (Coriat, 2009).

Como todo dispositivo de gobierno, la empresa flexible no es solo un haz de relaciones de poder cristalizadas en el esfuerzo de ciertas autoridades (gerentes, empresarios, grandes propietarios del capital, autoridades de gobierno) por desplegar técnicas y procedimientos particulares para dirigir la conducta de la fuerza de trabajo, sino que supone también, simultáneamente, ciertos juegos o discursos de verdad neomanageriales (respecto al trabajo, la empresa, la producción, la economía, los sindicatos, etc.) que hacen verosímiles y aceptables las nuevas prácticas de gobierno y gestión flexibles. Asimismo, se caracteriza por favorecer modalidades específicas de subjetivación y alentar ciertos modos de relación consigo mismo en los trabajadores, en el marco de los juegos de verdad de los discursos neomanageriales (Zangaro, 2011). Es, justamente, esa posibilidad de interrogar, ya no solo los modos de sujeción y objetivación de los trabajadores como sujetos laborales en ciertos diagramas de saber-poder de la empresa, sino también el modo en que los individuos -en el crisol de esos mismos juegos de verdad- se relacionan consigo mismos, se autogobiernan y ejercen sobre sí mismos singulares prácticas de sí, adquiriendo una experiencia concreta del mundo laboral y desplegando formas singulares de pensar y actuar respecto al trabajo, lo que constituye uno de los grandes aportes de la analítica de la gubernamentalidad al estudio de las subjetividades laborales en el nuevo capitalismo.

La perspectiva de la gubernamentalidad permite, entonces, interrogar los actuales espacios laborales de las grandes y modernizadas empresas de la región que han adoptado los principios flexibilizadores del nuevo management, como un campo de experiencia singular donde confluyen ciertas relaciones de poder, ciertos juegos de verdad y se promueven particulares modos de subjetivación, tres ámbitos estos que, como ya fue señalado, constituyen los ejes centrales de análisis desde la perspectiva de la gubernamentalidad, que deben ser estudiados considerando tanto su fuerte imbricación entre sí como su relativa especificidad e irreductibilidad (Castro-Gómez, 2011; Rabinow \& Rose, 2003).

\section{La empresa flexible y la racionalidad neomanagerial}

Destacamos cuatro aspectos de la racionalidad de gobierno de la empresa flexible que son especialmente relevantes para comprender las formas de subjetividad laboral que promueve, para visibilizar sus profundas resonancias con la gubernamentalidad neoliberal y la figura del empresario de sí, así como para ilustrar los aportes de la analítica de la gubernamentalidad a la comprensión de las transformaciones contemporáneas del trabajo. En cada una de estos aspectos se ponen en juego -con diferente énfasis- los tres ejes señalados (poder/ autoridad, verdad, subjetivación) ${ }^{6}$.

\section{Del asalariado al empresario de sí que invierte su capital humano}

Bajo las nuevas formas de gestión flexible, el trabajador ya no es representado ni interpelado principalmente como un individuo que arrienda su fuerza de trabajo a cambio de un salario para poder sobrevivir y que tiene intereses antagónicos (lo sepa o no) con el capital o la empresa. El trabajador es visto (y alentado a verse a sí mismo) como un empresario de sí que posee un particular capital inicial -su capital humano- el cual busca maximizar e invertir en diferentes inserciones laborales (Fleming, 2013; Weiskopf \& Munro, 2011). Se trata de un sujeto económico activo, que permanentemente calcula costos y beneficios, y entiende que al trabajar está haciendo una inversión de su propio capital (heredado y adquirido) de conocimientos, habilidades y

6 Como fue indicado lúcidamente por uno de los revisores anónimos, hubiese sido posible, y de algún modo deseable, organizar la exposición de esta sección con base en esos mismos tres ejes. Nos ha parecido, sin embargo, que organizar el argumento en cuatro aspectos diversos de la racionalidad de gobierno de la empresa flexible, en que confluyen de diversos modos los tres ejes, nos permite una aproximación, si bien menos sistemática, más dialogante con el campo de los estudios del trabajo y más fecunda de cara a comprender, desde distintos ángulos, el efecto en el ámbito de las subjetividades laborales de la empresa flexible. 
destrezas en pos de generar (al igual que cualquier empresario capitalista) algún tipo de ganancia o de renta (Castro-Gómez, 2011). Más que en términos de una relación laboral-fuertemente reglada, colectivizada, asimétrica y potencialmente conflictivaentre empresa y trabajadores, la empresa flexible vincula a la fuerza de trabajo (especialmente los cuadros profesionales) en términos de una alianza comercial, de duración incierta y beneficiosa para ambas partes, entre dos actores capitalistas (la empresa que posee los medios de producción y el trabajador que posee su capital humano) que están orientados a la maximización de su propio capital inicial. Como señala Read (2009), se deben entender los procesos de flexibilización del empleo, con su incremento de formas atípicas de contratación (temporales, jornada parcial, por prestación de servicios comerciales, etc.), no solo como una efectiva estrategia económica de las grandes empresas para reducir costos, sino como un importante vector de los procesos de producción de subjetividades laborales. Dicha lógica organizacional:

(...) alienta a los trabajadores a verse a sí mismos no como "trabajadores" en un sentido político, quienes tienen algo que ganar a través de la solidaridad y la organización colectiva, sino como "compañías de uno mismo". Ellos se convierten en individuos para quienes cada acción, desde tomar cursos de nuevas aplicaciones de software computacional hasta blanquearse los dientes, puede ser considerado como una inversión en capital humano. (p. 30)

Otros principios de gestión flexible a nivel del proceso de trabajo, tales como los sistemas de renta variable según productividad individual o la implementación de principios de competencia por bonos entre trabajadores de una misma unidad, producen también un entorno laboral que conduce la conducta de los trabajadores y anima a que ellos mismos se autogobiernen, en la lógica del "empresario de sí": sujetos responsables de sus éxitos y fracasos, para quienes la misma empresa es un mercado de alta competencia e incertidumbre que ofrece posibilidades para invertir el capital humano inicial y obtener una renta que lo maximice. Como escribió Foucault
(2007) en su análisis de las teorías del capital humano, la gubernamentalidad neoliberal, supone el proyecto de una sociedad como una red de unidades empresas y donde el individuo es llamado a verse a sí mismo como "un empresario de sí mismo, siendo para sí mismo su propio capital, siendo para sí mismo su propio productor, siendo para él mismo la fuente de sus rentas" (p. 232).

\section{La integración de la vida y la subjetividad social al proceso productivo}

Como ha analizado Peter Fleming (2013) a partir del concepto de "biocracia", un rasgo de la empresa flexible es la instrumentalización y utilización -como fuente para capitalizar- de atributos (no laborales) de la vida y la subjetividad social, los cuales fueron considerados como irrelevantes o incluso dañinos por la industria burocrática tayloristafordista (Perilleux, 2008).

En primer lugar, las nuevas formas de gestión alientan a los trabajadores a desplegar en el trabajo un conjunto de atributos personales (simpatía, sello personal, calidez, empatía) y a configurar redes colaborativas informales, los cuales aparecen como elementos que incrementan la productividad y la calidad de los desempeños. Más que prohibir verticalmente esas dimensiones más singulares, espontáneas e informales, la nueva empresa flexible las incita y promueve, en tanto mecanismos que juegan un rol crucial en la generación colectiva de innovación y conocimientos útiles, en la articulación de equipos y proyectos de trabajo, al igual que en la motivación y fuerte implicación de los sujetos con su trabajo, todo lo cual resulta un factor clave de la competitividad empresarial en el nuevo paradigma productivo (Coriat, 2009). De manera creciente, ese conjunto de destrezas subjetivas y habilidades blandas son consideradas en los procesos de selección de las grandes empresas, donde se estimula un perfil de trabajador capaz de incorporar sus habilidades interpersonales y su singularidad en procesos de trabajo cada vez más flexibles, fuertemente basados en el trabajo emocional y cognitivo, orientados a la calidad del servicio y la innovación continua y donde las lógicas de gestión fordistas basadas en la 
protocolización y estandarización de las conductas y en la vigilancia vertical se muestran ineficientes (Boltanski \& Chiapello, 2002; Neffa, 2003).

En segundo lugar, se observan lógicas empresariales que difuminan los límites entre tiempos, espacios y lógicas de trabajo y de no trabajo, desmantelándose, simbólica y prácticamente, la fuerte división vida/trabajo que operó como un principio central de la industria fordista y del liberalismo social. No se trata solo de que, producto de la intensificación del trabajo y las nuevas tecnologías de la información, el trabajo invade y ocupa otros tiempos de la vida social (personal, familiar, ocio, política, etc.). Tampoco, únicamente, de que producto de la flexibilización y precarización del empleo -con su creciente número de trabajadores con turnos variables, pluriactividad, empleo temporal y falta de acceso a derechos- se debilite la figura de una jornada laboral estandarizada y estable que separa en forma nítida y permanente los tiempos de trabajo de los tiempos de no trabajo (noche, el fin de semana, las vacaciones, períodos de enfermedad). Junto a lo anterior, la biocracia, en tanto diagrama de gobierno de la empresa flexible, considera muchas de las actividades no laborales como aportando a la esfera de la producción y la generación de valor (Fleming, 2013). Actividades tales como el viajar por el mundo, desarrollar actividades de voluntariado, hacer deporte, conocer los últimos juegos virtuales o series de TV, participar en encuentros con redes de exalumnos, tienen el potencial de aportar conocimientos, energías, redes, sintonía con los consumidores que generan valor en el marco de la racionalización productiva de la empresa flexible.

Este elemento de la racionalidad neomanagerial favorece procesos de subjetificación laboral productores de un sujeto que se representa y experimenta ya no como un asalariado que distingue claramente el tiempo y el espacio de trabajo, del tiempo y espacio de no trabajo, al igual que su rol y actividad laboral principalmente física y fuertemente prescrita, de su vida personal autónoma, de sus redes informales y de su singularidad cognitiva y emocional. Se trata, por el contrario, de un empresario de sí, abocado en cada espacio y minuto a la inversión y maximización de su capital humano, tanto por el anhelo de lograr una mayor capitalización y éxito a partir de la movilización de todos los aspectos de su existencia, como por el temor de que en un entorno laboral competitivo, incierto, cambiante y con débiles protecciones sociales, el que deja de capitalizar la totalidad de su vida y subjetividad puede quedar marginado, fuera de las redes productivas y del mercado laboral.

\section{De tecnologías disciplinarias de control a tecnologías de seguridad}

El espacio fabril bajo el capitalismo industrial, si bien inserto en el marco societal general de una gubernamentalidad de liberalismo social basada en mecanismos de seguridad, operó fuertemente a nivel local con base en tecnologías disciplinarias de control y gestión de la fuerza de trabajo. La empresa flexible, por el contrario, introduce en el corazón mismo del proceso productivo nuevas lógicas de gestión y control posdisciplinarias basadas en tecnologías de seguridad (Weiskopf \& Munro, 2011).

El nuevo management se aleja de la idea, propia de la disciplina fabril de la modernidad fordista, de que el trabajador es, en lo fundamental, un objeto, un mero cuerpo, que debe ser vigilado, controlado y corregido para volverlo dócil y acoplarlo útilmente a una maquinaria productiva previamente definida, planificada y establecida (Zangaro, 2011). Ya no se trata, en el tránsito de la figura de la fábrica fordista a la empresa flexible, de operar con una lógica disciplinar "centrípeta" que divide el espacio laboral, ubica los cuerpos, regula y estandariza los tiempos y los ritmos, prescribe cada una de las acciones por realizar, establece una norma, examina y vigila externamente la adecuación a la misma y sanciona a los trabajadores que se alejan de ella. Por el contrario, en el marco de la empresa flexible y sus lógicas de seguridad, se trata, como señalan Weiskopf y Munro (2011), de instalar una lógica "centrífuga" que permite la circulación, el movimiento, la iniciativa de los trabajadores. Se interviene ya no directa y prescriptivamente 
sobre sus acciones, sino sobre el medio ambiente de las mismas, sobre el campo y el marco posible de las acciones de los trabajadores, buscando que estos elijan autónoma y autorreguladamente determinados cursos de acción que favorecen la obtención de ciertos objetivos empresariales. Más que una normalización disciplinaria basada en la adecuación a una norma estándar, homogénea, única y previamente definida (normación), las formas de control de la empresa flexible operan con base en una normalización establecida a partir de la gestión de ciertos parámetros basados en análisis estadísticos que establecen contornos más amplios y móviles al interior de los cuales se espera actúen los trabajadores (Foucault, 2006). De este modo, la racionalidad de gobierno de la empresa flexible no supone ir contra la libertad de los trabajadores entendidos como empresarios de sí, sino influenciar y configurar el marco donde ellos toman sus decisiones y emprenden proyectos y acciones, así como ir gestionando los inevitables peligros y riegos que esa mayor autonomía de los trabajadores provoca continuamente en el dispositivo empresarial.

Lo anterior no implica, por cierto, que empíricamente en las empresas flexibles no sigan existiendo, especialmente para el caso de los trabajadores de baja calificación, fuertes mecanismos de control disciplinario; pero sí da cuenta, como señala Yáñez (2004), de que la racionalidad de gobierno del dispositivo apunta a un creciente uso de "formas internalizadas de autocontrol, auto-organización y autorracionalización por parte de los mismos trabajadores" (p. 70), las cuales resultan menos costosas y más eficientes a la luz de las demandas de versatilidad, innovación, cambio continuo y adaptabilidad estratégica a mercados competitivos e inciertos propias del capitalismo flexible. Se alientan, así, formas de subjetividad laboral caracterizadas por la alta iniciativa, capacidad de tomar decisiones y hacerse individualmente responsable de las mismas, habilidad para adaptarse a situaciones nuevas autónomamente, alta autorregulación, capacidad para operar en situaciones poco protocolizadas, fuerte autoexigencia para cumplir metas y acceder a los incentivos ofrecidos (Du Gay, 1996; Neffa, 2003).

\section{Emergencia de un nuevo telos o ideal de trabajador flexible}

Como señala Foucault (1996), un elemento central de la subjetivación es el telos o ideal de sujeto que da cuenta del tipo de persona en que uno aspira a convertirse a partir de un cierto trabajo sobre uno mismo. Para el caso de los procesos de subjetificación laboral en la empresa flexible, el discurso del new management ha jugado un rol central en instituir nuevas representaciones de lo que un sujeto debería aspirar a ser en tanto trabajador (Zangaro, 2011).

Frente al ideal fordista del operario disciplinado, anclado en una fuerte identidad de oficio y de clase, inserto en una tupida malla de protecciones sociales, proyectado en una trayectoria laboral relativamente estable e inserto en colectivos perdurables y homogéneos de trabajadores (la gran industria o el aparato público) que contribuyen al desarrollo nacional, habría ido emergiendo un nuevo ideal de trabajador emprendedor, flexible e individualizado. Se trata de un trabajador autoexigente, versátil, competitivo, autorregulado, polivalente, calculador, siempre disponible, eficiente, innovador, autónomo dentro de los márgenes establecidos, implicado física, cognitiva y afectivamente con la organización, leal y no conflictivo, apto para el trabajo en equipo, pero teniendo como valor último la proyección personal y la negociación individualizada con su empleador, capaz de adaptarse a los cambios y comprometido apasionadamente con la excelencia, la satisfacción del cliente (interno y externo) y el aprendizaje continuo (Boltanski \& Chiapello, 2002).

Este modelo e ideal de trabajador operaría crecientemente como un mandato social y como principio articulador de las subjetividades labores en la nueva empresa flexible, instituyendo una particular representación de lo que las personas deberíamos ser en tanto trabajadores, de las metas y anhelos que debemos perseguir, de las modalidades de relación con uno mismo y con los otros que debemos cultivar, de los principios de lo correcto y lo incorrecto a los que debemos adherir si queremos progresar, ser valorados y reconocidos en la empresa, el mercado laboral y en la vida social en general (Stecher, 
2009). Al mismo tiempo, como todo ideal de sujeto, este modelo de trabajador supone un recorte de sentido que crea y requiere un dominio de alteridad, otras figuras del trabajador (el burócrata, el obrero con conciencia de clase, el apegado a una identidad de oficio claramente delimitada, etc.) que son tipificadas como aquello que se aleja del mandato social, como aquel "otro" que debe ser rechazado por arcaico, irracional, falto de sentido, anormal, perturbador del orden y contra el cual se afirma y sostiene el ideal hegemónico del nuevo trabajador emprendedor y flexible (Butler, 1997; Portocarrero, 2001).

\section{Consideraciones finales}

A partir de la descripción de estos cuatro ejes se ha buscado dar cuenta de algunos elementos claves de la racionalidad de gobierno de la empresa flexible, destacando el modo como esta promueve y alienta particulares modalidades de subjetividad laboral en el crisol de una trama de técnicas, saberes y prácticas. Estas formas de subjetividad tienen fuertes resonancias con lo descrito previamente respecto a la gubernamentalidad neoliberal y la figura del empresario de sí, y deben ser comprendidas no solo como un efecto posterior al funcionamiento de la empresa flexible, sino como una condición de posibilidad y sustentabilidad de esa misma racionalidad de gobierno que la produce. Como se ha destacado, la empresa flexible debe ser entendida, antes que como un símbolo del debilitamiento de la disciplina fabril y el paso a un mundo laboral emancipado y sin relaciones de poder, como un dispositivo que articula nuevas modalidades de regulación y control de la fuerza de trabajo y de producción de subjetividades laborales, como expresión de un nuevo arte de gobierno del trabajo que conlleva nuevos peligros, lo que exige, como diría Foucault, la atención del pensamiento crítico (Fleming, 2013; Rabinow \& Rose, 2003).

Para finalizar, se presentan dos consideraciones relativas al uso de la analítica de la gubernamentalidad en la investigación empírica sobre trabajo y subjetividad en América Latina. En primer lugar, es importante no tomar la descripción de la em- presa flexible (un tipo ideal), su racionalidad de gobierno y de los modos de subjetividad laboral que promueve como una descripción empírica que daría cuenta de la complejidad de los espacios de trabajo de las grandes empresas y de los sujetos laborales efectivamente existentes. Lo que se ha descrito $-y$ ese suele ser el énfasis en los trabajo de Foucault- es una lógica y racionalidad de gobierno empresarial, cuya caracterización permite formular hipótesis y preguntas directrices fecundas para la investigación empírica en grandes empresas y con sujetos laborales específicos, pero en ningún caso reemplaza dicho trabajo de investigación. Como indicara Foucault (2003), toda racionalidad de gobierno es siempre una racionalidad, histórica y situada, que falla, que es resistida y refractada, que nunca se completa. La analítica de la gubernamentalidad pone el foco en un análisis de la dimensión programática y estratégica de una racionalidad de gobierno, la cual una vez puesta en práctica nunca funciona como fue planeada, no logra abarcar integralmente el campo donde se despliega, tiene efectos no previstos e incluso entra en tensión con otras racionalidades circulantes o debe acoplarse con otras lógicas con las que se contaminan mutuamente (Dean, 2008; Miller \& Rose, 2008). Al respecto, es posible recordar el carácter híbrido de los procesos de reestructuración productiva en muchas de las grandes empresas en América Latina, donde se observa en ocasiones la coexistencia, en una misma unidad empresarial y en función de los diferentes grupos ocupacionales, de lógicas de racionalización productiva taylorista-fordista y lógicas neomanageriales y flexibles, e incluso de lógicas tradicionales o artesanales (De la Garza, 2000).

Lógicas de racionalización productiva heterogéneas cuya operación, a su vez, no puede ser entendida, si no se analiza el modo como se acoplan, en muchos contextos laborales de la región, con otras lógicas de gobierno de la población basadas en la racialización o generización de importantes segmentos de la fuerza de trabajo. Se plantea, así, el desafío de desarrollar investigación empírica en los escenarios concretos de trabajo de las grandes y modernizadas empresas de la región, sin olvidar que dichas empresas son solo un fragmento de los 
heterogéneos y desiguales mundos del trabajo en América Latina, donde las lógicas de autosubsistencia y trabajo informal, y de modernización (neo)taylorista-fordista con sus vigorosas lógicas de disciplinamiento y control individualizado de los cuerpos, sigue siendo el marco de experiencia laboral para una gran parte de los sujetos que trabajan.

En segundo lugar, es importante recordar que la analítica de la gubernamentalidad no supone en ningún caso, la afirmación de un poder que elimina totalmente las posibilidades de resistencia a los dispositivos de gobierno. Por el contrario, exige siempre en la investigación empírica, el análisis de las distintas formas de contra-conducta, subversión o resistencia que se establecen en todo campo de conducción de la conducta y que abren, a partir del a-sujetamiento a nuevos juegos de verdad y del despliegue de nuevas técnicas de sí, posibilidades de otras subjetividades y modos de existencia (Lemke, 2010). El análisis de la gubernamentalidad es también siempre el de formas de agenciamiento (individual y colectivo) que politizan un escenario dado a partir de la afirmación del derecho a ser diferente y a no ser gobernado de ese modo, y abren un espacio de problematización crítica del presente que ilumina los límites históricos y contingente de lo que somos y alumbra nuevas posibilidades de vida (Foucault, 2003). Como escribiera Foucault en ¿Qué es la Crítica?, dando cuenta de la centralidad de la articulación entre poder, verdad y subjetividad como clave para interrogar críticamente nuestra actualidad, sus formas de gobierno y sus líneas de fuga y desestabilización:

El corazón de la crítica está básicamente hecho de [y orientado a] el haz de relaciones que articulan (...) el poder, la verdad y el sujeto. Si la gubernamentalización es en verdad este movimiento por el cual se trataba, en la realidad misma de una práctica social, de sujetar a los individuos [de producirlos como un particular tipo de sujeto] a través de unos mecanismos de poder que invocan una verdad, pues bien, yo diría que la crítica es el movimiento por el cual el sujeto se atribuye el derecho de interrogar a la verdad acerca de sus efectos de poder y al poder acerca de sus discursos de verdad. Entonces la crí- tica sería el arte de la voluntaria insubordinación, de una indocilidad reflejada. La crítica aseguraría el des-asujetamiento del sujeto en el contexto de lo que nosotros podríamos llamar, en una palabra, las políticas de verdad. (p. 266)

Se espera haber contribuido a visibilizar el potencial de la perspectiva de la gubernamentalidad para analizar en América Latina los juegos de verdad, las relaciones de poder y los vectores de subjetivación que caracterizan a la nueva empresa flexible, cuya comprensión es central para la tarea del pensamiento crítico, para alentar nuevas formas de agenciamiento individual y colectivo e imaginar otros modos de habitar y relacionarse con el trabajo en el mundo contemporáneo.

\section{Referencias}

Amin, A. (1994). Post-fordism: A reader (studies in urban and social change). Malden, MA: Blackwell.

Battistini, O. (Comp.). (2004). El trabajo frente al espejo. Continuidades y rupturas en los procesos de construcción identitaria de los trabajadores. Buenos Aires: Prometeo Libros.

Binkley, S. (2014). Happiness as enterprise: An essay on neoliberal life. Nueva York: SUNY Press.

Boltanski, L., \& Chiapello, E. (2002). El nuevo espíritu del capitalismo. Madrid: Akal.

Burchell, G. (1996). Liberal government and techniques of the self. En A. Barry, T. Osborne \& N. Rose (Eds.), Foucault and Political Reason. Londres: UCL Press.

Butler, J. (1997). The psychic life of power: Theories in subjection. Palo Alto, CA: Stanford University Press.

Castells, M. (2001). La era de la información (Vols. 1 y 2). Madrid: Alianza.

Castells, M. (2005). Globalización, desarrollo y democracia: Chile en el contexto mundial. Santiago: Fondo de Cultura Económica.

Castro, E. (2011). Diccionario Foucault. Temas, conceptos y autores. Buenos Aires: Siglo XXI.

Castro-Gómez, S. (2010). Historia de la gubernamentalidad. Razón de Estado, liberalismo y neoliberalismo en Michel Foucault. Bogotá: Siglo del Hombre Editores. 
Coriat, B. (2009). Pensar al revés. Trabajo y organización en la empresa japonesa. México: Siglo XXI.

De la Garza, E. (Coord.). (2000). Tratado latinoamericano de sociología del trabajo. México: Fondo de Cultura Económica.

Dean, M. (2008). Governmentality: Power and rule in modern society. Londres: Sage.

Dreyfus, H., \& Rabinow, P. (2001). Michel Foucault: más allá del estructuralismo y la hermenéutica. Buenos Aires: Nueva Visión.

Du Gay, P. (1996). Consumption and identity at work. Londres: Sage.

Fleming, P. (2013). When 'life itself' goes to work: Reviewing shifts in organizational life through the lens of biopower. Human Relations, 67(7), 875-901.

Foucault, M. (1996). El yo minimalista y otras conversaciones. Buenos Aires: Biblioteca de la Mirada.

Foucault, M. (2001). El sujeto y el poder. En H. Dreyfus \& P. Rabinow (Eds.), Michel Foucault: más allá del estructuralismo y la hermenéutica (pp. 241-259). Buenos Aires: Nueva Visión.

Foucault, M. (2001). What is critique? En P. Rabinow $\&$ N. Rose (Eds.), The essential Foucault. Nueva York: The New Press.

Foucault, M. (2006). Seguridad, territorio, población. Curso en el Collège de France (1977-1978). Buenos Aires: Fondo de Cultura Económica.

Foucault, M. (2007). El Nacimiento de la biopolítica. Curso en el Collège de France (1978-1979). Buenos Aires: Fondo de Cultura Económica.

Harvey, D. (1998). La condición de la posmodernidad. Barcelona: Amorrortu.

Lemke, T. (2010). Los riesgos de la seguridad. Liberalismo, biopolítica y miedo. En V. Lemm (Edit.) Michel Foucault: Neoliberalismo y Biopolítica. Santiago:UDP ediciones.

McNay, L. (2009). Self as enterprise: Dilemmas of control and resistance in Foucault's The Birth of Bipolitics. Theory, Culture ES Society, 26(6), 55-77.

Miller, P., \& Rose, N. (2008). Governing the present. Cambridge: Polity Press.

Neffa, J. (2003). El trabajo humano. Contribuciones al estudio de un valor que permanece. Buenos Aires: Lumen.

Perilleux, T. (2008). Las transformaciones contemporáneas del trabajo. Nuevas canteras de pensam- iento y de acción. En B. Espinosa (Coord.) Mundos del trabajo: pluralidad y transformaciones contemporáneas. Quito: FLACSO.

Portocarrero, G. (2001). Nuevos modelos de identidad en la sociedad peruana. En G. Portocarrero \& J. Komadina (Eds.), Modelos de identidad y sentidos de pertenencia en Perú y Bolivia. Lima: Instituto de Estudios Peruanos.

Rabinow, P \& Rose, N. (2001). (Edit.) The Essential Foucault. New York: The New Press.

Ramos, C. (2009). La transformación de la empresa chilena. Santiago de Chile: Universidad Alberto Hurtado.

Ramos, C. (2012). El ensamblaje de ciencia social y sociedad. Santiago: Universidad Alberto Hurtado.

Read, J. (2009). A genealogy of homo-economicus: Neoliberalism and the production of subjectivity. Foucault Studies, 6, 25-36.

Rose, N. (2003). Identidad, genealogía e historia. En S. Hall \& P. Du Gay (Comps.), Cuestiones de identidad cultural (pp. 214-250). Buenos Aires: Amorrortu.

Rose, N. (1997). El gobierno de las democracias liberales avanzadas: del liberalismo al neoliberalismo. Archipiélago, 29, 25-40.

Rose, N., \& Miller, P. (1992). Political power beyond the state: Problematics of government. British Journal of Sociology, 43, 173-205.

Senellart, M. (2006). Situación de los cursos. En M. Foucault. Seguridad, territorio, población: Curso en el Collège de France (1977-1978). Buenos Aires: Fondo de Cultura Económica.

Sennett, R. (2006). La cultura del nuevo capitalismo. Barcelona: Anagrama.

Soto, A. (Ed.). (2008). Flexibilidad laboral y subjetividades. Santiago de Chile: Universidad Alberto Hurtado.

Stecher, A. (2009). La competencia por el éxito y la búsqueda de la autenticidad. Modelos de identidad en el Chile actual. Psicoperspectivas, 7(2), 266-292.

Stecher, A. (2013). Un modelo crítico-interpretativo para el estudio de las identidades laborales. Contribuciones a la investigación psicosocial sobre trabajo y subjetividad en América Latina. Universitas Psychologica, 12(4), 1311-1324.

Stecher, A., \& Godoy, L. (2014). Transformaciones del trabajo, subjetividad e identidades. Lecturas psicoso- 
ciales desde Chile y América Latina. Santiago: Ril Editores.

Tittoni, J., \& Nardi, E. (2011). Sujetividade e trabalho. En L. Holzmann \& A. Cattani (Orgs.), Dicionário de Trabalho e tecnología (pp. 375-378). Porto Alegre: Zouk Editora.

Tokman, V. (2011). Informalidad en América Latina. Balance y perspectivas de política. Revista Internacional de Estadística y Geografía, 2(3), 16-31.

Vázquez, F. (2005). Empresarios de nosotros mismos. Biopolítica, mercado y soberanía en la gubernamentalidad neoliberal. En J. Ugarte (Comp.), La administración de la vida. Barcelona: Anthropos.
Vallas, S. P. (1999). Rethinking post-Fordism: The meaning of workplace flexibility? Sociological Theory, 17(1), 68-101.

Wagner, P. (1997). Sociología de la modernidad. Barcelona: Herder.

Weiskopf, R., \& Munro, I. (2011). When 'life itself' goes to work: Reviewing shifts in HRM. Organization, 19(6), 685-702.

Zangaro, M. (2011). Subjetividad y trabajo. Una lectura foucaultiana del management. Buenos Aires: Herramienta. 